\title{
DIAGRAMAS DE INTERACCIÓN AXIL-FLEXIÓN- CORTANTE PARA PERFILES METÁLICOS EN DOBLE T
}

\author{
(INTERACTION GRAPHS FOR STEEL H-SECTIONS SUBJECTED TO BENDING, SHEAR AND \\ AXIAL FORCES)
}

Ramón Irles Más, Dr. I.C.C.P., Prof. Tit. Univ.

Dpto. Ing. de la Construcción, O. P. e Inf. Urbana

Francisco Irles Más, I.T.O.P., Prof. Tit. E.U.

Dpto. Expresión Gráfica y Cartografía

Univ. de Alicante

ESPAÑA

\section{RESUMEN}

El uso de los diagramas de interacción como herramienta de predimensionado o dimensionado de secciones de piezas prismáticas sometidas a combinaciones de varios esfuerzos constituye algo habitual en la técnica. Desde hace tiempo se dispone de colecciones de estos diagramas para las diversas tecnologias: hormigón armado, acero estructural y secciones mixtas, bajo diversas solicitaciones.

En este articulo se expone minuciosamente el planteamiento general para la obtención de los diagramas correspondientes a flexocompresión simple de perfiles con sección transversal en doble T. Se realiza un estudio general de la superficie limite de interacción de los tres esfuerzos, describiendo detalladamente sus tres regiones, y la generación de los diagramas de interacción que corresponden a sus secciones por valores de axil constante.

\section{SUMMARY}

Interaction graphs are extensively used as a tool for designing prismatic member sections subjected to several combined stresses. For a long time, sets of these graphs are available in building technologies: reinforced concrete, steel and composite sections, under various stresses.

This paper shows the general formulation for obtainning the graphs corresponding to bending, shear and axial forces of $H$-shaped steel sections. A general study of these stresses interaction limit surface is made, including a detailed description of its three regions, and the generation of interaction graphs corresponding to their sections by constant axial values.

\section{Introducción}

La interacción entre tensiones normales y tangenciales que expresa el criterio de plastificación de Misses, aplicada al punto más solicitado de una sección metálica suele considerarse como límite para el dimensionado o predimensionado del elemento resistente.
La expresión de las tensiones que en función de los esfuerzos proporciona la Resistencia de Materiales, permite aplicar dicha condición límite directamente a ellos. Dada la forma de tal criterio, las superficies límite de interacción para ternas de esfuerzos aplicados resultan ser planos, cuádricas, superficies más complejas o una mixtura de ellas. 
En dos artículos precedentes [1], [2] se expuso la obtención de los diagramas de interacción (curvas de nivel de la superficie límite, según valores escalonados de alguno de los esfuerzos) para secciones metálicas huecas bajo varias combinaciones de esfuerzos (véase también [4] y [5]).

En el presente, se expone la obtención de la superficie límite de interacción entre axil, flector y cortante, para las secciones metálicas en doble T (series IPN, IPE, HEB, HEA, HEM) y los diagramas correspondientes a sus secciones por planos de esfuerzo axil constante.

\section{Planteamiento del problema}

En la figura 1 se representan la notación, los esfuerzos considerados y las tensiones generadas en la sección doble $\mathrm{T}$. Dichas tensiones se expresan:

$$
\begin{aligned}
& \sigma=\frac{N}{A}+\frac{M}{I} y=v+\mu y \\
& \tau=\frac{Q}{I} \frac{M_{e s t}}{e}=\left[\begin{array}{l}
q \cdot a(s) \text { en el ala } \\
q \cdot\left[b-\frac{1}{2} y^{2}\right] \text { en el alma }
\end{array}\right.
\end{aligned}
$$

siendo:

$$
\begin{aligned}
& v=\frac{\mathrm{N}}{\mathrm{A}} \\
& \mu=\frac{\mathrm{M}}{\mathrm{I}} \\
& \mathrm{q}=\frac{\mathrm{Q}}{\mathrm{I}} \\
& \mathrm{a}(\mathrm{s})=\left(\mathrm{h}-\mathrm{e}_{\mathrm{a}}\right) \frac{\mathrm{S}}{2} \\
& \mathrm{~b}=\frac{\mathrm{S}_{\mathrm{x}}}{\mathrm{e}_{\mathrm{h}}} \\
& \mathrm{S}_{\mathrm{x}}=\text { Momento estático de media sección, respec- } \\
& \text { to del eje } \mathrm{x} . \\
& \mathrm{I}=\text { Inercia de la sección, respecto del eje } \mathrm{x} .
\end{aligned}
$$

Con el criterio de Misses, el cuadrado de la tensión de comparación será:

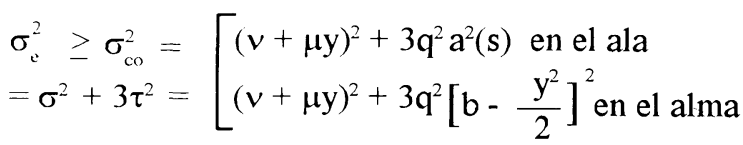

\section{Búsqueda del punto más solicitado}

3.1) EN EL ALA. El punto más solicitado del ala es el definido por $s=b$ ', $y=h / 2$ (fig. 1 ), en el que se produce un máximo absoluto de $\sigma^{2}$ co y cuando éste sea el más solicitado de la sección, la condición límite de agotamiento será:
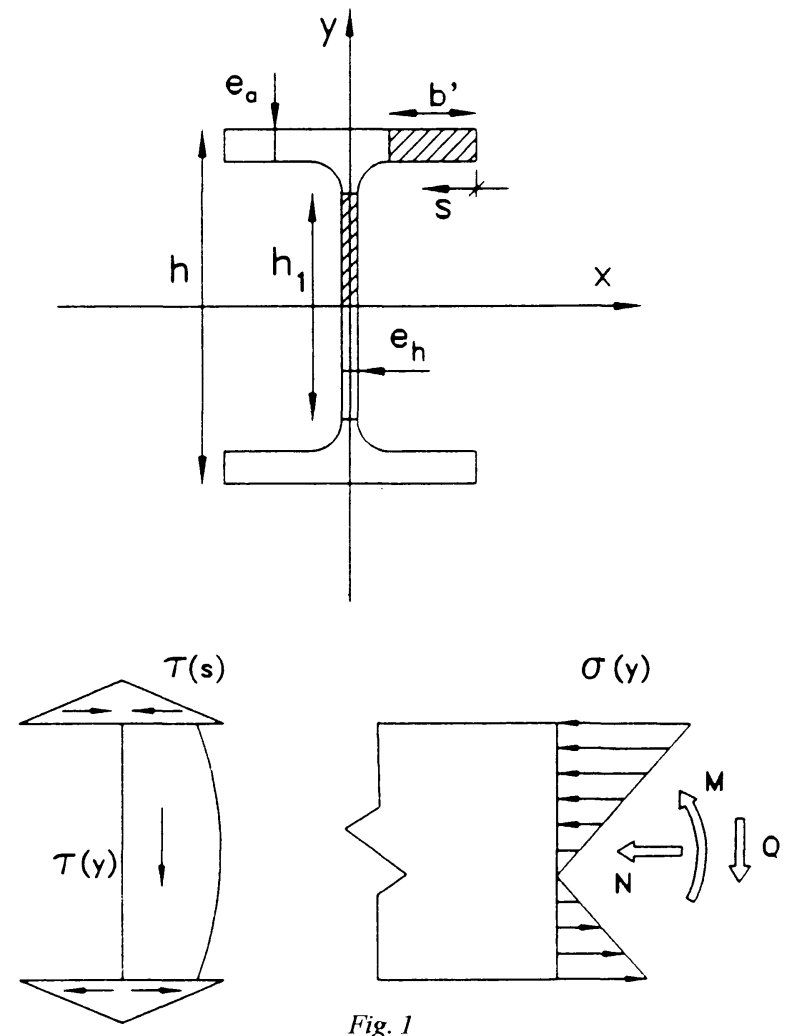

Fig. 1

$\sigma_{\mathrm{e}}^{2}=v^{2}+2 \frac{\mathrm{h}}{2} \nu \mu+\frac{\mathrm{h}^{2}}{4} \mu^{2}+3 \mathrm{q}^{2} \mathrm{a}_{1}^{2}$

siendo:

$$
a_{1}=a\left(b^{\prime}\right)=\left(h-e_{a}\right) \cdot \frac{b^{\prime}}{2}
$$

3.2) EN EL ALMA. Las funciones $\sigma, \tau, \sigma^{2}, \tau^{2}$ y $\sigma^{2}$ $=\mathrm{f}(\mathrm{y})$ en el alma se representan en la figura 2 , expresándose ésta última:

$$
f(y)=v^{2}+2 v \mu y+\mu^{2} y^{2}+3 q^{2}\left(b^{2}-b y^{2}+\frac{y^{4}}{4}\right)
$$

pudiéndose producir su mayor valor, bien en un máximo absoluto en $y=h_{1} / 2$, o bien en uno relativo en cualquier posición y $\left[0, h_{1} / 2\right]^{1}$, que pasamos a estudiar, anulando su derivada $1^{\mathrm{a}}$.

$$
f^{\prime}(y)=2 v \mu+2 \mu^{2} y+3 q^{2}\left(y^{3}-2 b y\right)=0
$$

Fuera del plano $\mathrm{q}=0=\mathrm{Q}$, donde el diagrama de interacción es, de forma inmediata, la recta

$$
\sigma_{\mathrm{e}}=\frac{\mathrm{N}}{\mathrm{A}}+\frac{\mathrm{M}}{\mathrm{W}}(\mathrm{W}=\text { Módulo resistente de eje fuerte })
$$

la condición requerida es:

$$
y^{3}+\frac{2}{3 q^{2}}\left(\mu^{2}-3 b q^{2}\right) y+\frac{2 v \mu}{3 q^{2}}=0
$$

\footnotetext{
${ }^{1}$ Se puede restringir a este intervalo el problema, dada la simètria de $\tau^{2}$
} 

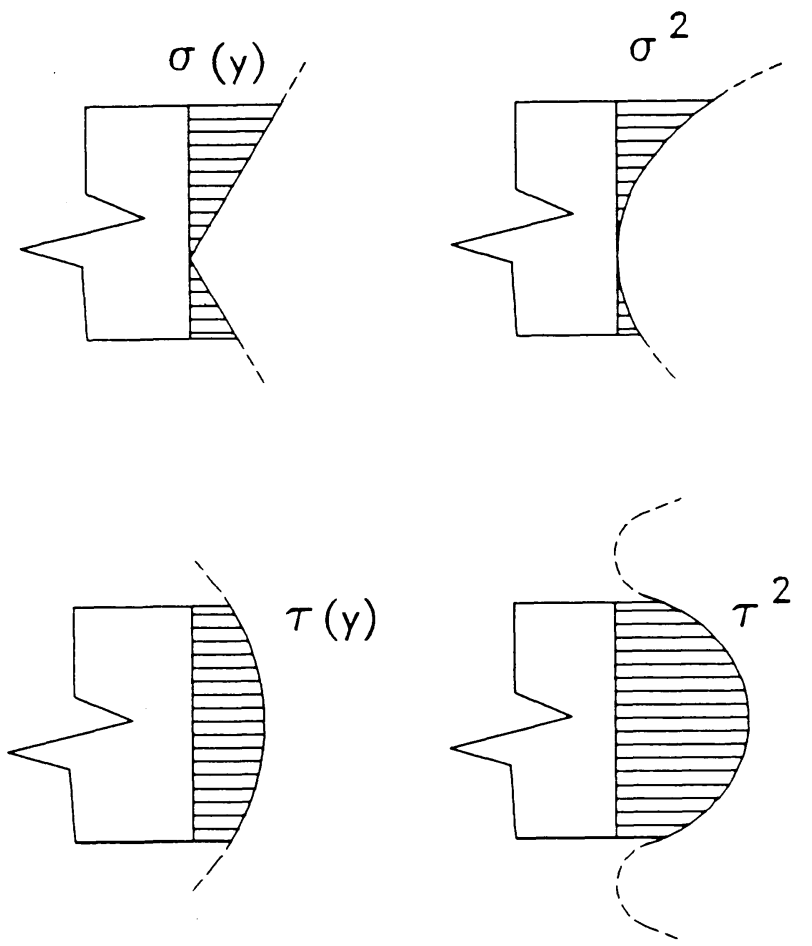

Fig. 2

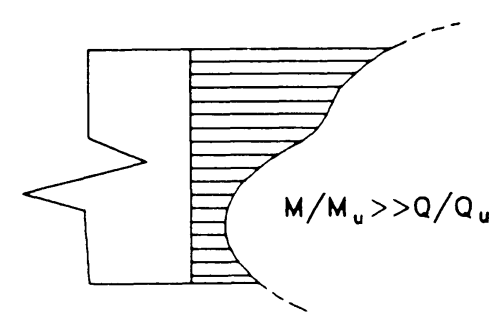

$\sigma_{c o}^{2}=\sigma^{2}+3 \tau^{2}$

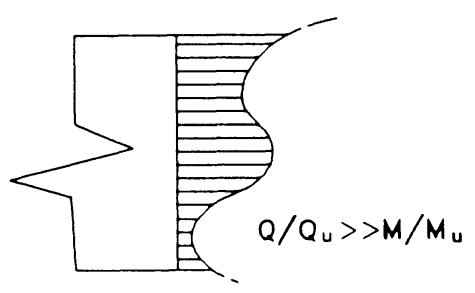

ecuación polinómica de grado 3, cuyas soluciones son:

$$
\left.3 b q^{2}-\mu^{2}\right)>0
$$

$$
y_{1}=\frac{\sqrt{8\left(3 b q^{2}-\mu^{2}\right)}}{3 q} \cos \frac{\theta}{3}
$$

$$
y_{2}=\frac{\sqrt{8\left(3 b q^{2}-\mu^{2}\right)}}{3 q} \cos \left[\frac{\theta}{3}+\frac{2 \pi}{3}\right]
$$

$$
y_{3}=\frac{\sqrt{8\left(3 b q^{2}-\mu^{2}\right)}}{3 q} \cos \left[\frac{\theta}{3}+\frac{4 \pi}{3}\right]
$$

$$
\text { siendo } \theta=\operatorname{arcos} \frac{-9 v \mu q}{\sqrt{8\left(3 b q^{2}-\mu^{2}\right)^{3}}} \in\left[\pi, \frac{3 \pi}{2}\right]
$$

En el plano $v=0=\mathrm{N}$ desaparece el término independiente de (5) y las soluciones se simplifican a:

$$
\begin{gathered}
y_{1}=0 \\
y_{2,3}= \pm \sqrt{\frac{2\left(3 b q^{2}-\mu^{2}\right)}{3 q^{2}}}
\end{gathered}
$$

Entre las soluciones de (6) siempre existe una real; para que las otras dos lo sean también, deberá ocurrir simultáneamente $\frac{9 v \mu q}{\sqrt{8\left(3 b q^{2}-\mu^{2}\right)^{3}}}<1-81 v^{2} \mu^{2} q^{2}-8\left(3 b q^{2}-\mu^{2}\right)^{3}<0$

En realidad el cumplimiento de (8.a) queda garantizado con el de (8.b), por tanto la condición se reduce a ésta última.

$\mathrm{El}$ análisis de la segunda derivada de la función $\mathrm{f}$ :

$$
f^{\prime \prime}(y)=9 q^{2} y^{2}-2\left(3 b q^{2}-\mu^{2}\right)
$$

muestra que la condición (8.a) es necesaria para la existencia de un máximo relativo, pero sólo es suficiente en el plano $v=0$, donde en $\mathrm{y}_{1}=0$ se presenta un máximo relativo y en $\mathrm{y}_{2}, \mathrm{y}_{3}$ mínimos relativos.

En general, la existencia de máximo requiere que:

$$
9 q^{2} y_{1}^{2}-2\left(3 b q^{2}-\mu^{2}\right)<0
$$

Sustituyendo $y_{1}$ de (6.a), recordando la relación trigonométrica $\cos \theta=4 \cos ^{3} \theta / 3-3 \cos \theta / 3$,y tras algunas manipulaciones algebraicas, esta expresión se convierte idénticamente en la (8.b); lo cual es lógico, dáda la forma de la función $\mathrm{f}$ :

-Si sólo se anula su $1^{\text {a }}$ derivada en un punto real, se trata de 
un mínimo relativo pues $\mathrm{f}(\mathrm{y})$ es continua e indefinidamente derivable, y creciente para $y \rightarrow \pm \infty$

-Si se anula en tres puntos reales, uno es máxim. :' los otros dos mínimos relativos, por la misma razón, y r c no puede comprobarse sustituyendo $y_{2}$ e $y_{3}$ en (9). En este caso, el máximo relativo siempre es la solución intermedia

$$
\text { que es } y_{1} \text { si en (7) se elige } \theta \in\left[\pi, \frac{3 \pi}{2}\right]
$$

Pero, en realidad, la existencia del máximo relativo sólo será condición limitante cuando éste:

- Se encuentre dentro del alma:

$$
\mathrm{y}_{1}<\frac{\mathrm{h}_{1}}{2}
$$

Sustituyendo $y_{1}$ de (6.a), recordando la expresión trigonométrica citada, y tras algunas. manipulaciones algebraicas, esta condición se expresa:

$$
8 h_{1} \mu^{2}+16 v \mu+3 h_{1} q^{2}\left(h_{1}{ }^{2}-8 b\right)<0
$$

- Supere al valor de f en el extremo de la misma:

$$
f\left(y_{1}\right)>f\left(h_{1} / 2\right)
$$

es decir,

$$
\mu_{2}\left[\mathrm{y}_{1}+\frac{\mathrm{h}_{1}}{2}\right]+2 v \mu+3 \mathrm{q}^{2}\left[\mathrm{y}_{1}+\frac{\mathrm{h}_{1}}{2}\right]\left[\frac{1}{4}\left[\mathrm{y}_{1}^{2}+\frac{\mathrm{h}_{1}^{2}}{4}\right]-\mathrm{b}\right]<0
$$

- Y supere al valor de $\sigma^{2}$ en el ala:

o sea:

$\mu_{2}^{2}\left[\frac{h^{2}}{4}-y_{1}^{2}\right]+2 v \mu\left[\frac{h}{2}-y_{1}\right]+3 q^{2}\left[a_{1}{ }^{2}-\left[b-\frac{y_{1}{ }^{2}}{2}\right]^{2}\right]<0$

La racionalización de (12) y (13), teniendo en cuenta la expresión de $\mathrm{y}_{1}$, es excesivamente larga y conduce a expresiones de grado elevadísimo en $q, \mu, \mathrm{y} v$, por lo que no se explicitará.

3.2.1) Cuando el máximo absoluto es el valor $\mathrm{f}\left(\mathrm{h}_{1} / 2\right)$, la condición se expresa:

$$
v^{2}+2 v \mu \frac{h_{1}}{2}+\mu^{2} \frac{h_{1}^{2}}{4}+3 q^{2} a_{2}^{2}-\sigma_{e}^{2}=0
$$

siendo $a_{2}{ }^{2}=\left[b-\frac{h_{1}^{2}}{8}\right]^{2}$

Ésta será la aplicación sólo cuando dicho máximo absoluto supere al de la tensión de comparación del ala:

$$
\mu^{2}\left[\frac{h^{2}}{4}-\frac{h_{1}^{2}}{4}\right]+2 v \mu\left[\frac{h}{2}-\frac{h_{1}}{2}\right]-3 q^{2} a_{3}^{2}<0
$$

siendo $\mathrm{a}_{3}^{2}=\mathrm{a}_{2}^{2}-\mathrm{a}_{1}^{2}>0$

3.2.2) Finalmente, cuando el máximo es relativo en $y_{1}$, la condición límite de agotamiento será:

$$
\left(v+\mu y_{1}\right)^{2}+3 q^{2}\left[b-\frac{y_{1}^{2}}{2}\right]^{2}-\sigma_{e}^{2}=0
$$

resultando igualmente inviable su racionalización en términos de $\mathrm{q}, \mu, \mathrm{y} v$, habida cuenta de la expresión de $\mathrm{y}_{1}$.

\section{Interpretación geométrica y discusión completa del problema.}

Las expresiones (3), (14) y (16) constituyen las condiciones límite de interacción Cortante-Flector-Axil, y en un espacio $q-\mu-\nu$ (ó $\mathrm{Q}, \mathrm{M}, \mathrm{N}$ ) representan las ecuaciones de tres superficies. Cada una de ellas es de aplicación, como hemos visto, en unos dominios de dicho espacio delimitados por las superficies que corresponden a las ecuaciones de las expresiones (8b), (11), (12), (13) y (15) puestas en el límite de la igualdad a cero. A continuación, pasamos a estudiar dichas superficies.

\section{1) MÁXIMO EN EL ALA}

\subsection{1) Dominio}

El dominio de validez de la superficie (3) es la región del espacio $q-\mu-\nu$ complementaria de la dada por la condición (15); en el límite:

$$
\mu^{2}\left[\frac{h^{2}}{4}-\frac{h_{1}^{2}}{4}\right]+2 v \mu\left[\frac{h}{2}-\frac{h_{1}}{2}\right]-3 q^{2} a_{3}^{2}<0
$$

El estudio de esta superficie cuádrica [3] manifiesta que se trata de un cono de vértice el origen, de eje contenido en los cuadrantes impares del plano $q=0$ y de directriz elíptica, tangente al plano $\mu=0$ a lo largo del eje Ov, (fig.3). El dominio de aplicación de (3) es el interior del cono que denominamos $\mathrm{CO} 1$.

\subsection{2) Superficie limite}

La superficie límite (3) es otra cuádrica; su estudio [3] manifiesta que se trata de un cilındro cuyo eje está igualmente contenido en el plano $q=0$ y pasa por el origen, y cuya directriz es también elíptica (fig.3). Lo denominaremos CI1

\section{2) MÁXIMO EN EXTREMO DEL ALMA}

\subsection{1) Dominio}

El dominio de aplicación de la superficie límite (14) viene dado por (15) es decir, la región del espacio exterior al cono CO1 (fig. 3 ) pero limitada por las condiciones (8b), (11), (12) y (13). 


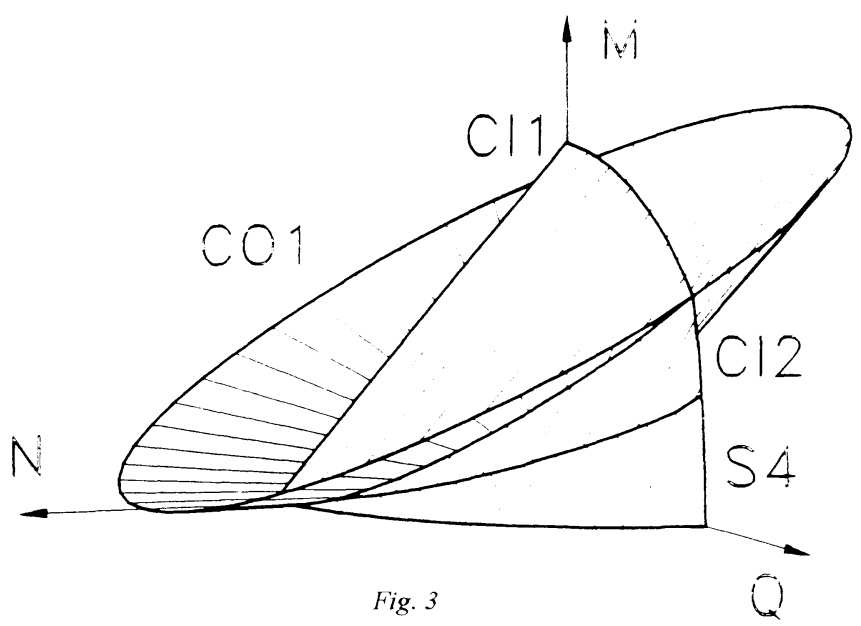

La condición (8b), en su límite, representa la ecuación de una superficie de orden superior, que denominaremos S1, cuyo estudio revela que:

- Es tangente al plano $v=0$ a lo largo de su recta $\mu=\mathrm{q} \sqrt{3 \mathrm{~b}}$.

- Es tangente al plano $\mu=0$ a lo largo del eje Ov.

- Es cóncava hacia el eje $\mu+$ en el primer octante.

La condición (11), en su límite, representa una cuádrica que resulta ser también un cono, que denominaremos $\mathrm{CO} 2$, de las mismas características que $\mathrm{CO} 1, \mathrm{y}$ tangente al mismo a lo largo de Ov.

La condición (12), en el límite, representa la ecuación de una superficie de orden superior que denominaremos S2, de las mismas características que la S1, tangente a ella a lo largo de Ov, pero que corta al plano

$\nu=0$ en la recta $\mu=q \sqrt{3 b-3 \frac{h_{1}^{2}}{16}}$, de menor pendiente.

La condición (13), en su límite, representa igualmente una superficie de orden superior que denominaremos S3, similar a las S1 y S2, que corta al

plano $v=0$ en la recta $\mu=\frac{2}{h} q \sqrt{3 b^{2}-3 a^{2}}$

Sólo para valores de $\mu$ por encima de alguna de las superficies $\mathrm{S} 1, \mathrm{~S} 2$ o S3, o dentro del cono $\mathrm{CO} 2$ y simultáneamente fuera del cono $\mathrm{CO} 1$, es de aplicación la superficie límite de interacción (14).

\subsection{2) Superficie limite}

Dicha superficie (14) es también una cuádrica. Se trata de otro cilindro elíptico que denominaremos $\mathrm{CI} 2$ (fig. 3) de características similares al CI1, cuyo eje pasa, igualmente, por el origen pero es de menor pendiente, y presenta distintas excentricidades

(c) Consejo Superior de Investigaciones Científicas Licencia Creative Commons 3.0 España (by-nc)
Por la curva alabeada, intersección de ambos cilindros, pasa también el cono $\mathrm{CO} 1$ que delimita sus dominios de validez

\section{3) MÁXIMO INTERIOR AL ALMA}

\subsection{1) Dominio}

El dominio de aplicación de la tercera porción de la superficie límite está constituido por la región inferior a las superficies S1, S2 y S3, y exterior al cono CO2 simultáneamente.

\subsection{2) Superficie limite}

La superficie de interacción (16), que denominaremos S4, en este caso es también de orden muy elevado, y (fig. 3):

- Corta al plano $v=0$ a lo largo de la recta $q=q_{11}=\frac{\sigma_{c} e_{n}}{\sqrt{3} S}$

- Corta al plano $\mu=0$ a lo largo de la elipse $v^{2}+3 q^{2} b^{2}=\sigma^{2}$.

\section{Representación de la superficie límite. Diagramas de interacción.}

La superficie límite está, pues, constituida por tres porciones de dos cilindros elípticos y una superficie de orden superior, secantes entre sí. Dada la configuración de los dominios de validez de cada una de estas porciones, y las características de éstas, los diagramas de interacción más convenientes son los producidos por secciones de las superficies con planos de axil constante $(\nu=$ cte. ); de esta forma, todos los diagramas presentan un tramo de curva de cada superficie, en su correspondiente dominio, mientras que las otras dos alternativas $\mu$ cte. ó $q$ cte. presentan tipologías distintas al no cortar todos los planos a las tres superficies.

En la fig. 4 se esquematiza el proceso de obtención de estos diagramas y en las 5 y 6 se representan los correspondien-

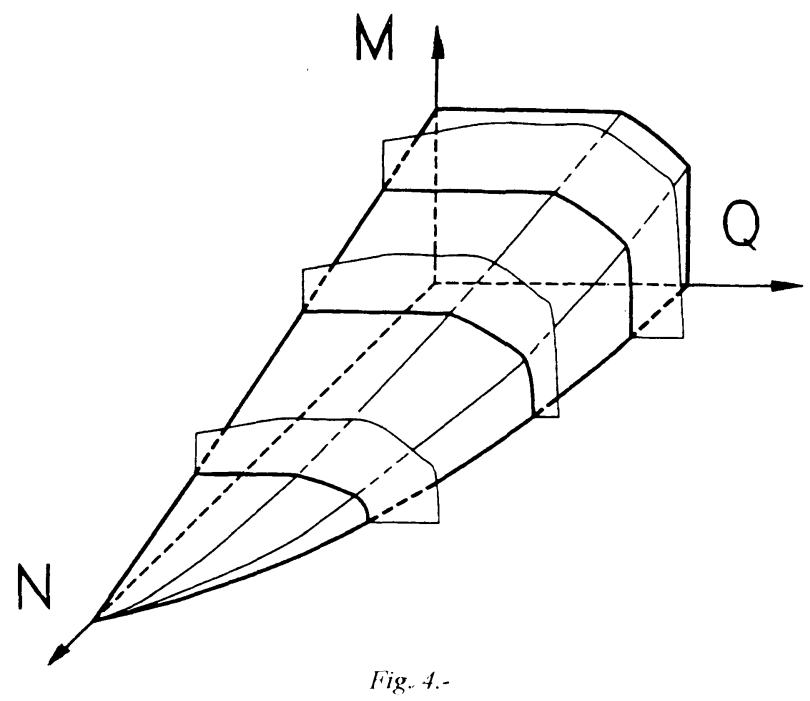




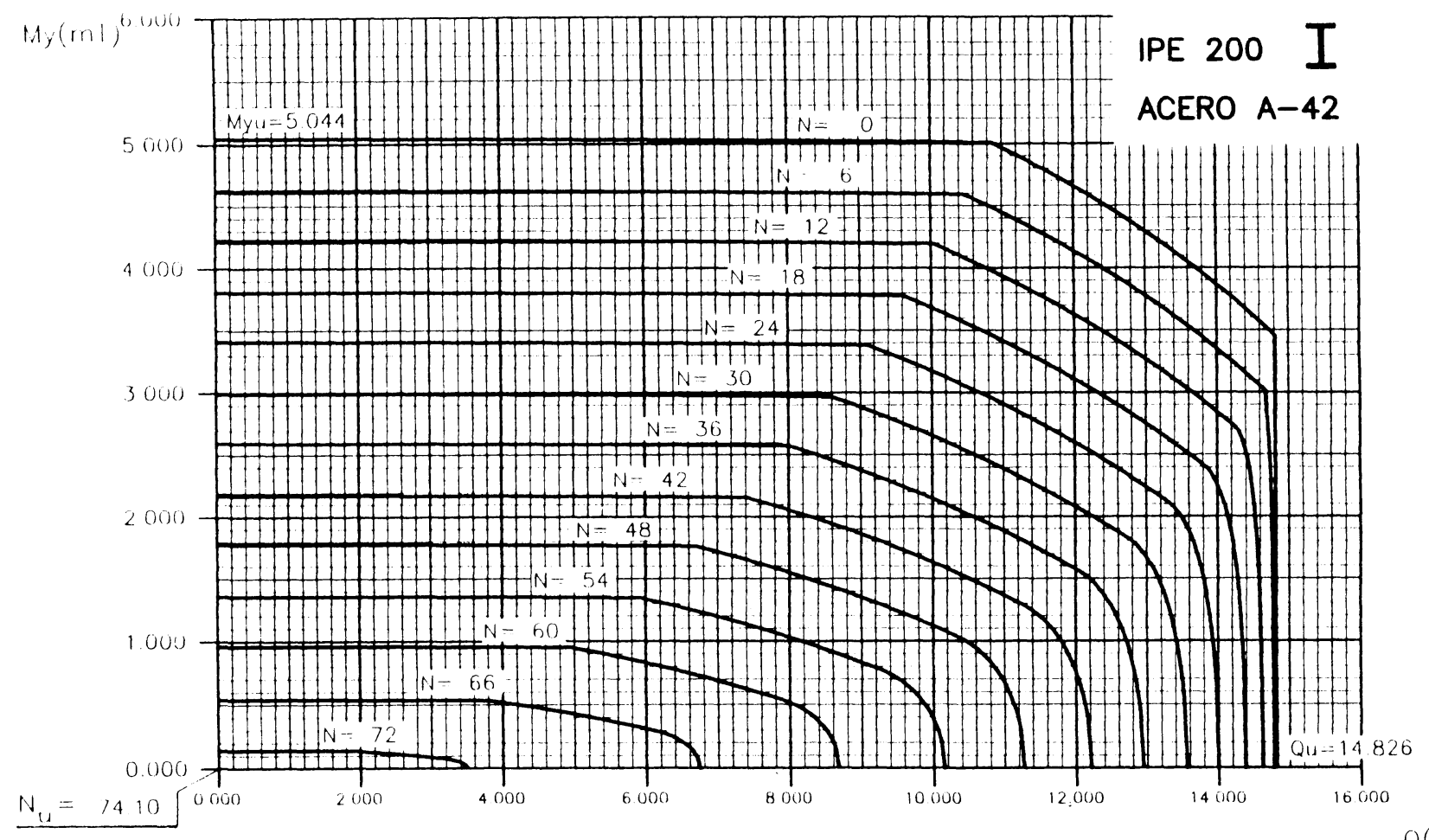

Fig. 5

My $(m)$

HEB 200 I

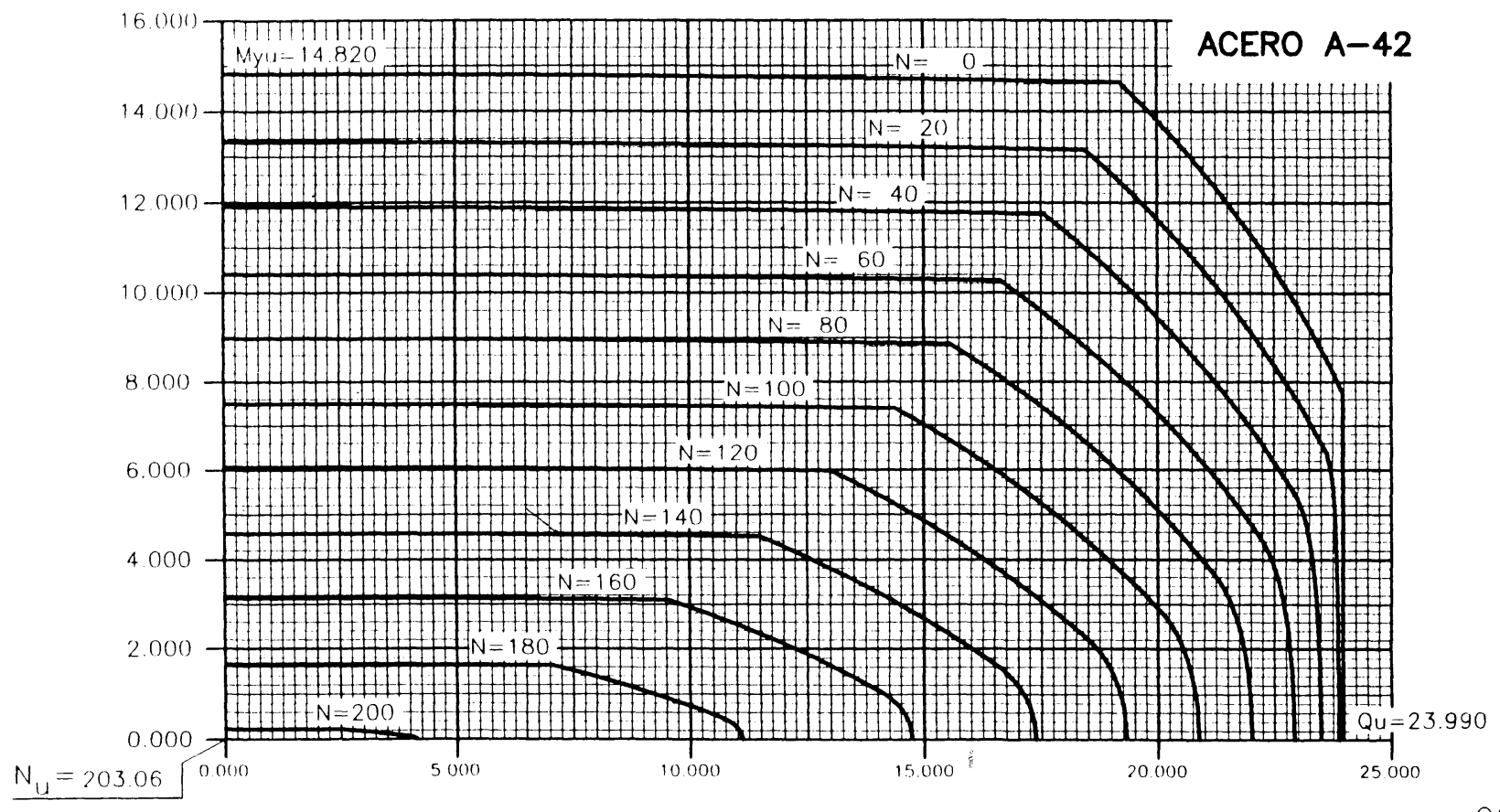

Fig. 6 
tes a un perfil IPE 200 y a un HEB 200, respectivamente, preparados para su uso.

\section{Conclusión}

Se ha expuesto la formulación analítica para la obtención de la superficie límite de interacción de los esfuerzos axil, flector de eje mayor y cortante concomitante, en perfiles metálicos de sección doble $\mathrm{T}$.

Esta superficie está compuesta por tres regiones, dos de ellas cuádricas y la tercera de orden superior, delimitados sus dominios de validez en el espacio N-M-Q, igualmente por superficies cuádricas y de orden superior.

Una vez obtenidas y estudiadas las superficies, se han obtenido los diagramas de interacción que corresponden a secciones por esfuerzo axil constante, que resultan las más convenientes, resultando trazos mixtilíneos de dos tramos elípticos y otro de orden superior, con discontinuidad de derivada en los puntos de cambio.

Finalmente, como ejemplo, se han representado los diagramas correspondientes a un perfil doble $\mathrm{T}$ de ala estrecha y otro de ala ancha, de las series comerciales.

\section{BIBLIOGRAFÍA}

[1] IRLES MÁS, R., IRLES MÁs, F. Y CARRATALÁ CLIMENT, D.: Diagramas de interacción para secciones metálicas huecas a flexión esviada compuesta, Informes de la Construcción, 46, nº 431, Páginas 41 a 48, 1994.

[2] IRLES MÁS, R., IRLES MÁS, F. Y CARRATALÁ CLIMENT, D.: Diagramas de interacción para secciones metálicas circulares a flexotorsión simple, Informes de la Construcción, 46, n 432, Páginas 33 a 40, 1994.

[3] J. GARCÍA GARCÍA Y M. LÓPEZ PELLICER: Álgebra lineal y Geometria, Marfil, Alcoy. $4^{\text {a }}$ Ed. 1984.

[4] T. ATSUTA Y W.F. CHEN: Theory of beam columns, MacGraw-Hill B.C., 1976.

[5] C.E.N.: Eurocódigo 3. Art ${ }^{o}$. 5.4.7, C.E.N., Bruselas, 1992.

\section{Publicación del Instituto Eduardo Torroja - CSIC}

\section{JORNADAS SOBRE CEMENTO ALUMINOSO}

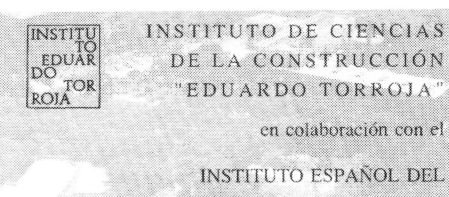

CEMENTO Y SUS APLICACIONES IECA

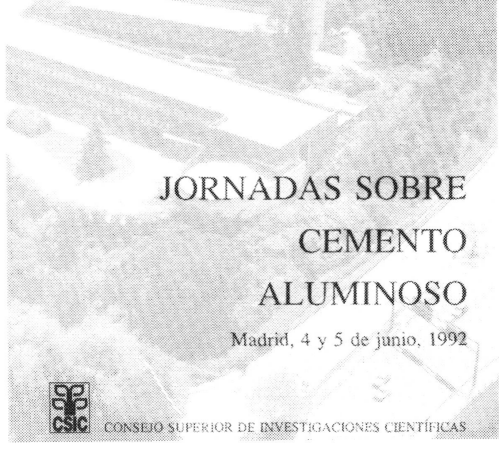

El Instituto Eduardo Torroja publica íntegramente -en colaboración con el Instituto Español del Cemento y sus Aplicaciones (IECA) - las “Jornadas sobre cemento aluminoso", celebradas en junio de 1992.

Se recogen las conferencias dictadas por destacados especialistas en la materia, así como las intervenciones suscitadas en los coloquios y en la interesante Mesa Redonda, tras la cual se clausuraron las Jornadas.

El tratamiento dado al tema del hormigón y cemento aluminoso por los intervinientes abarca tanto aspectos rigurosos científico-técnicos como de índole práctica, abordando temas de corrosión de armaduras, durabilidad, protección y reparación de los elementos constructivos con daños causados por diversas motivaciones ampliamente expuestas.

Este libro será, sin duda, una eficaz ayuda tanto para el científico o el técnico estudioso de esta materia, como para aquellos profesionales interesados en un tema de tanta importancia y actualidad como es el relacionado con los hormigones de cemento aluminoso.

Un volumen de $15,5 \times 22,5 \mathrm{~cm}, 186$ págs., con figuras de línea, gráficos, tablas, etc. 